Brain Inspired Cognitive Systems

August 29 - September 1, 2004

University of Stirling, Scotland, UK

\title{
DIPOLE SOURCE LOCALISATION USING INDEPENDENT COMPONENT ANALYSIS: SINGLE TRIAL LOCALISATION OF LASER EVOKED PAIN
}

\author{
Thang Nam Hoang ${ }^{1}$, Wael El-Deredy ${ }^{1}$, Deborah E. Bentley ${ }^{2}$, Anthony K.P. Jones ${ }^{2}$, Paulo J. \\ Lisboa $^{1}$ and Francis Mcglone ${ }^{3}$ \\ ${ }^{1}$ School of Computing and Mathematical Sciences, Liverpool John Moores University, Byrom \\ Street, Liverpool L3 3AF, UK \\ ${ }^{2}$ University of Manchester Rheumatic Diseases Centre, Hope Hospital, Salford M6 8HD, UK \\ ${ }^{3}$ Centre for Cognitive Neuroscience, University of Wales, Bangor LL57 2DG, UK
}

\section{Correspondent: Thang Nam Hoang}

Tel: 44-(0)-151-231-2188, Email: hoangnamthang@yahoo.co.jp

\begin{abstract}
The accuracy of the inverse solution that finds the spatial location of the generating sources from averaged scalp-recorded event related potentials (ERPs) relies on assumptions about the ERP signals and the sources. We provide evidence that using independent component analysis (ICA) as a signal decomposition filter prior to applying the inverse solution reveals sources that cannot be detected by conventional source localisation methods.
\end{abstract}

Five clusters of sources emerged: a single source cluster in caudal cingulate and bilateral sources in secondary somatosensory cortex (SII), inferior parietal cortex, premotor cortex and insular cortex. The locations of the source dipoles were consistent with findings using $f$ MRI and PET but have not all been previously detected in a single electrophysiological study. In addition, the timecourse of the activation of these dipoles was estimated.

The results suggest that using ICA to localise single trial data is a powerful tool for exploring the spatiotemporal dynamics of rapid and complex brain processes.

\section{INTRODUCTION}

In standard electrical dipole source localisation, averaged event related potentials (ERPs) are used as a 'noise free' version of ERP. On the basis of a priori knowledge of the neurophysiology and/or analysis of the variance data, a decision is made whether the ERP peak under investigation is likely to be the result of single dipole or multiple dipole sources, for which the inverse solution aims to find the best fit.

This modelling method has two problems. The first problem is the validity of the use of averaged ERPs. The single trial ERPs have been considered as resulted from discrete, functionally defined neural activity. Therefore, the averaged ERPs have been considered as the noise free version of single trial ERPs, since averaging progress have removed the background EEG. However, recently, works by (Basar et al., 2001; Jung et al., 2001; Makeig et al., 2002) show that the single trial ERPs are likely to result from phase oscillation of ongoing EEG. Therefore averaged ERPs are not noise free version of single trial ERPs, but may in fact introduce artefacts (see Hoang et al, BICS 2004, submitted).

The second problem is that the inverse solution for the multiple dipole sources has been shown to be not as reliable as inverse solution for single dipole source(Cuffin, 1998; Mosher et al., 1993). If the ERP peak is due to a single-dipole source, the solution is only affected by the accuracy of the head model parameters but not by the fitting method, whereas the solution for a peak due to multiple sources is dependent on the accuracy of the assumption about the number of sources (Wang, 1994), which is more ambiguous. In particular, the pain process is unlikely to have multiple sources which do not have overlapping activation, therefore the accuracy of single source dipole inverse solution in this case is questionable.

Jung et al (2001), Makeig et al (2002) and Zhukov et al (2000) have suggested using independent component analysis (ICA) to separate the mixture of generating sources in the ERP signals into independent components. The inverse solution for a single source dipole can then be applied to each of the components to estimate their spatial locations. The advantage of this technique is that it uses single trial ERPs signals without any averaging, and avoids the problems associated with the inverse solution for multiple source dipoles. 
In this paper, we apply ICA methods to source localisation for the single trial laser evoked pain potentials (LEPs) to localise the brain source dipoles related to pain process. LEP is ERP recorded from laser stimuli, which evoke the heat pain response on the skin.

Neuroimaging studies of pain process in laser evoked heat pain using PET and fMRI have suggested the involvement of a number of cortical areas: Insula, Primary and Secondary Somatosensory (SII), Inferior Parietal, Premotor and mid-cingulate or caudal anterior Cingulate cortices. (Coghill et al., 1999; Derbyshire, 2000; Derbyshire et al., 1997; Lotze et al., 2001; Niddam et al., 2002; Peyron et al., 2000).

In addition, source localization studies of EEG and MEG from multi-channel LEP recordings have also been used to identify possible cerebral generators of pain-related signals (Bentley et al., 2003; Frot et al., 2001; Peyron et al., 2002; Watanabe et al., 1998) (Ninomiya et al., 2001) (Bromm and Lorenz, 1998; Kanda et al., 2000; Nakamura et al., 2002; Tarkka and Treede, 1993). These have shown sources in SII, insula and mid cingulate. However, these studies have not revealed the full extent of the sources in the same way that PET and $f$ MRI studies have.

Hence it is difficult to interpret the relationship between the estimated sources from electrophysiological (LEP, MEP) studies and activated brain region in $f$ MRI and PET as well as inferring the functionality of these regions.

In this study, the result of source localisation using ICA shows five clusters of source dipoles, which clearly identified in the caudal cingulate, secondary somatosensory (SII), inferior parietal, insular and pre-motor cortices, consistent with results from fMRI and PET in other research(Coghill et al., 1999; Derbyshire et al., 1997; Lotze et al., 2001; Niddam et al., 2002).

\section{MATERIALS AND METHODS}

\section{Laser stimulation and data acquisition}

The study was approved by the Local Research Ethics Committee. Data were recorded from one right-handed female volunteer, aged 30 years, who gave her informed consent. She was in good health and not taking medication at the time of the study. Throughout the experiment, the subject wore protective goggles for safety and earplugs to mask acoustic interference from the laser.

The study comprised 3 recording sessions held at approximately the same time on different days. Each session consisted of 3 LEP recordings using the same methodology. During each recording, $60 \mathrm{CO}_{2}$ laser stimuli (100 ms pulse duration, $15 \mathrm{~mm}$ beam diameter) were delivered to the right dorsal forearm at $10 \mathrm{~s}$ intervals. Stimuli were randomly moved around a $5 \times 3 \mathrm{~cm}$ area (positioned relative to individual anatomical characteristics for accurate reproduction) to avoid habituation/sensitization and possible skin damage.

The subject rated each stimulus on a $0-10$ scale for pain intensity, approximately $3 \mathrm{~s}$ after the stimulus. Stimuli were kept at an intensity that the subject rated as moderately painful (i.e. $6-7$ on the $0-10$ scale).

Late LEPs were recorded from 64 scalp electrodes (positioned according to the 10-20 system), referenced to linked earlobes (QuikCap system, NeuroScan, Inc.). EEG data were sampled at a rate of $500 \mathrm{~Hz}$, with a gain of 500, and band pass filters of $0.15-30 \mathrm{~Hz}$ (SynAmps, NeuroScan, Inc.). The vertical and horizontal electrooculogram was also recorded for the purposes of ocular artefact reduction. The impedance of all electrodes was below $5 \mathrm{k} \Omega$. At the end of each LEP recording session, a digitiser (Polhemus 'Fastrak') was used to encode the electrode positions, which are later used to co-register the ERP with the structural MRI of the brain of the same subject (see Bentley et al for details). The structural MRI was acquired on a different day to the LEP recordings (1 Tesla Siemens scanner, T1-weighted image, 256×256 pixels, each $0.94 \times 0.94 \mathrm{~mm}, 108$ sagittal slices, each $1.67 \mathrm{~mm}$ thick).

\section{Data pre-processing}

Continuous EEG data were corrected for ocular artefact prior to epoching. A $200 \mathrm{~ms}$ pre-stimulus interval was used for baseline correction. Epochs with further artefact and not rated as painful (i.e. less than 4 on the $0-10$ scale) were rejected. The accepted epochs of each block then concatenated together. Therefore, the data using in this paper will be 9 matrixes from 9 blocks of experiment.

\section{Concatenation of single trial ERPs}

The purpose of ERPs source localisation problem is to reconstruct the brain electrical sources, which generated the ERPs of certain stimulus. The single trial ERPs of the same stimulus are concatenated before applying ICA to do blind source separation. The continuous EEG may contain the signal from the sources, which are not active in ERPs time windows.

Source separation process using informax ICA algorithm(Bell and Senowski, 1995) was applied to the 64 channels of each concatenated LEP signal of each of the nine experimental blocks, decomposing the signal into 64 maximally independent 
components. Each independent component, now a partial signal of the original recording, was averaged across the trial in the same block.

Theoretically, applying ICA to each single trial ERPs separately is possible. However, applied ICA to each single trial ERPs will generate larger number of ICs, which is not practical to compute the inverse solution for each single extracted ICs. By concatenated single trial ERPs, it is possible to reduce the number of extracted IC, and do not reduce the accuracy of source localisation if the ERPs of the same stimulus are generated from similar brain domains.

\section{Independent components selecting method}

Assume that we want to do source localisation from $M$ channels of single trial ERPs data. Source separation process using informax ICA algorithm(Bell and Senowski, 1995) first applied to this $M$ channels of each concatenated ERPs signal, decomposing the signal into $M$ maximally independent components. The projection of each component back onto the electrodes, now a partial signal of the original recording, was averaged across the trial in the same block. The derived averaged independent components then ordered by their averaged activation power by following formula:

$$
P\left(I C_{k}\right)=\sum_{i=1}^{M} \sum_{j=1}^{N}\left[V_{i}^{I C_{k}}\left(t_{j}\right)\right]^{2}
$$

where $P\left(I C_{k}\right)$ is the averaged activation power of the $k^{\text {th }}$ IC. $V_{i}^{I C_{k}}\left(t_{j}\right)$ is the voltage of averaged $I C_{k}$ at electrode $i$ at sampling timing $t_{j} . N$ is the number of data point $(N=$ sampling frequency $\times$ length of time windows of single trial ERP).

In each block, we have $M$ ICs, therefore the total averaged power of all ICs in each block is: $\sum_{k=1}^{64} P\left(I C_{k}\right)$.

The following step is to select the ICs, which corresponded to $80 \%$ contribution to this total averaged power. By this method, the selected IC is the ICs which have more activation after averaging, and therefore more likely relevant to laser stimuli. Source localisation then be applied to each selected IC to calculate the location of the dipole source.

Base on this power criteria, from $9 \times 64$ ICs, 180 ICs which have dominant power on total averaged power of each block are selected for the inverse solution. By this method, the selected IC is the ICs which have more activation after averaging.

\section{Source localisation}

Source localization analysis was performed by CURRY® ${ }^{\circledR}$ 4.5 software (Compumedics USA Ltd) on realistic head model, which was rebuilt by the module of the same software from MRI images of the same subject. The source localisation was applied on entire data window (from 0 to $1000 \mathrm{~ms}$ ) of averaged IC. Electrodes location using in the head model was measured at each experiment block by digitizer. Because of the nature of the ICA, the activation of extracted IC is statistically independent from each other; hence, it is reasonable to assume that, each IC is generated by single dipole source or synchronised bilateral dipole sources. Therefore, for each IC, two mode of source localization are used:

(i) Source localization for single dipole source

(ii) Source localization for bilateral dipole source

The results with smaller error are selected. In case, where the solution for single dipole source gives a smaller error, but the location of that source is not physiologically relevant (in the area which not likely to be EEG source like white matter area), then the bilateral dipole source result are selected. Any result with variance (error) larger than $20 \%$ are consider as noise.

\section{RESULTS}

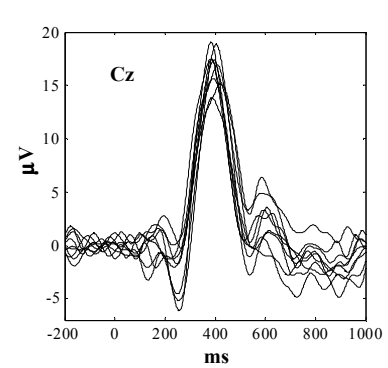

(a)

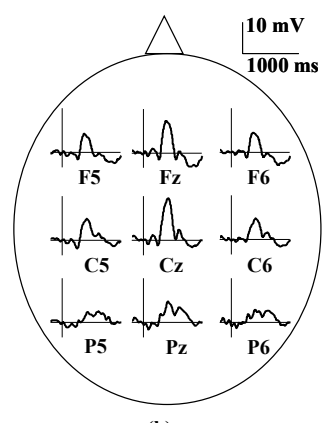

(b)
Figure 1: Examples of averaged LEP signal. (a) Averaged LEP signal at $\mathrm{Cz}$ from 9 data sets. (b) Example of averaged LEP signal of one data set at 9 groups of electrodes. The LEP signals have a positive peak between $350 \sim 450 \mathrm{~ms}$. The peak appears in most electrodes around the head shows the largescale integration of the LEP signal.

The examples of averaged LEPs at $\mathrm{Cz}$ from 9 data sets are showed in Figure 1.a. The LEP signals have a positive peak between $350 \sim 450 \mathrm{~ms}$. The peak appears in most electrodes around the head shows the large-scale integration of the LEP signal (see Figure 1.b) 

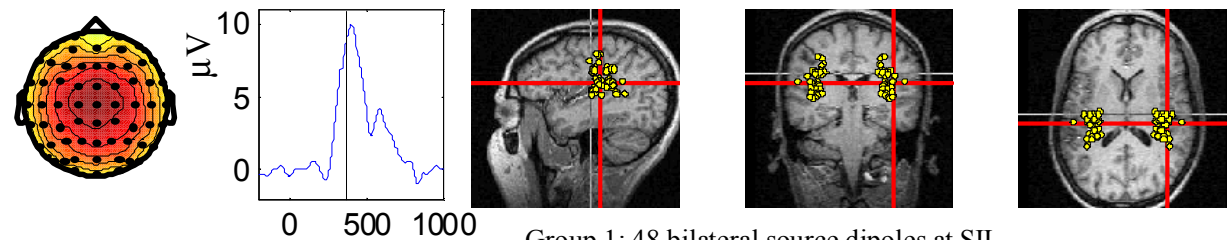

Group 1: 48 bilateral source dipoles at SII
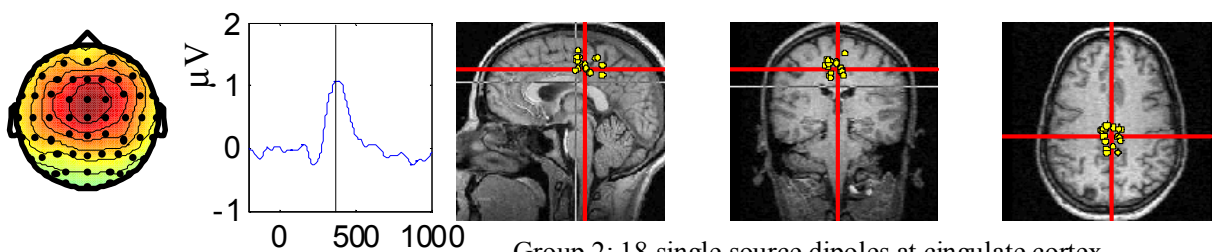

Group 2: 18 single source dipoles at cingulate cortex
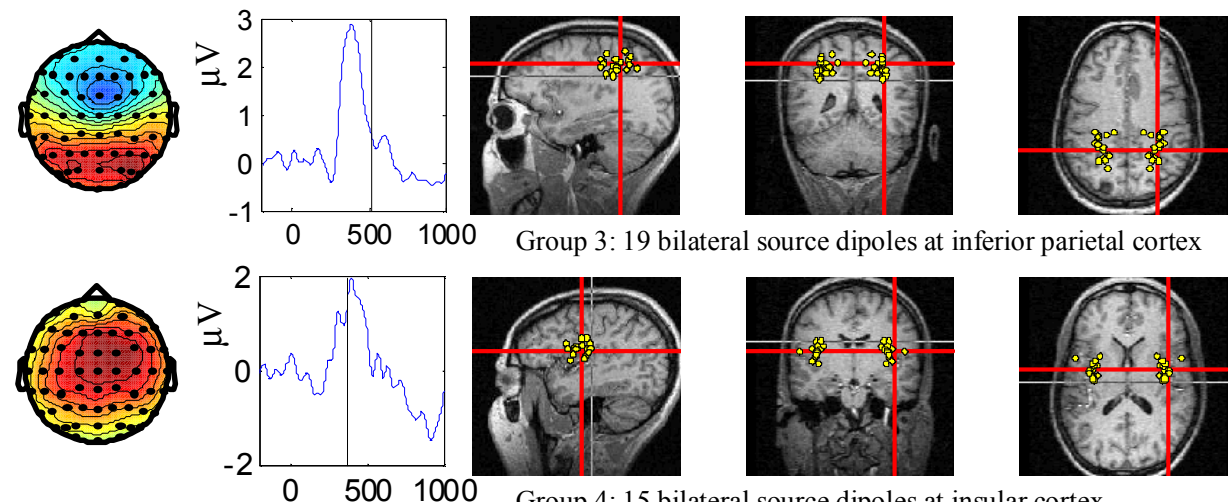

Group 4: 15 bilateral source dipoles at insular cortex
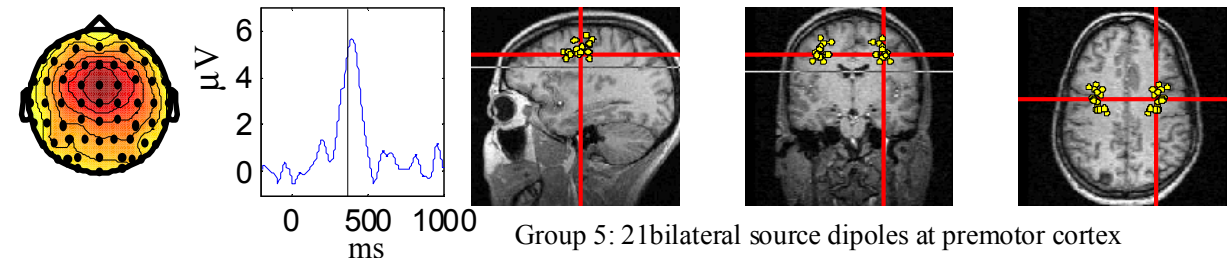

Figure 2: Clusters of dipole sources in separate brain region, their averaged activation course and corresponded topographic map. First column shows the averaged topographic maps of each source group, at the timing marked by strait line in the graph on the second column. Second column shows the averaged activation of each source group. Three right columns show the detail of source on MRI slides. Each group of source dipoles include the dipole from all 9 data sets, except group 6 (primary cortex) only have dipole from 4 data sets.

From 180 ICs, 30 single dipole sources and 114 bilateral dipole sources were reconstructed. The remaining 36 ICs were considered as noise, since the error in source localisation calculation of these ICs were larger than $20 \%$, or their locations were outside of the brain. Errors of the analysis were relatively small since the source localisation was applied for entire time window of averaged ICs (Figure 3).

The dipoles were classified by their anatomical location (Figure 2). The dipoles then were clustered by their anatomical location verified by brain atlas (Mai et al., 1997). From each data block, 5 clusters of dipoles were found. These clusters are located in SII, cingulated cortex, inferior parietal cortex, insular cortex, premotor cortex.

\section{DISCUSSION}

The brain responses at SII in laser pain stimuli are reported in (Lotze et al., 2001; Niddam et al., 2002) using fMRI, in (Coghill et al., 1999) using PET. The responses in insula were reported in (Peyron et al., 2002) using $f$ MRI, PET as well as LEP source dipole localisation, in (Coghill et al., 1999) (Niddam et al., 2002) using PET. The brain responses at premotor cortex in laser pain stimuli are reported in (Derbyshire et al., 1997; Niddam et al., 2002) using PET. The responses in cingulate cortex are reported in (Coghill et al., 1999)(PET). The responses at inferior parietal cortex are reported in (Coghill et al., 1999; Derbyshire et al., 1997) (PET). Although some of the previous study using LEP source localisation have detected the brain source at SII, insular and cingulated cortex(Bentley et al., 2003; Frot et al., 2001; Peyron et 
al., 2002), this study is the first time have localised all these sources in the same experiment.
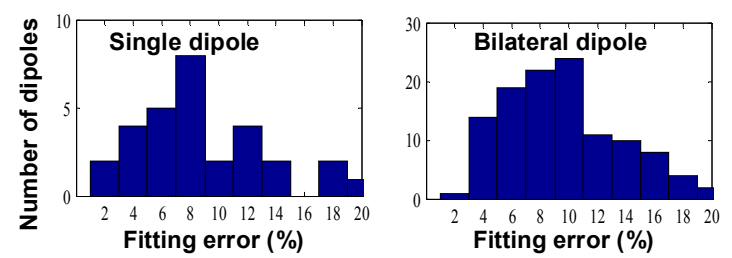

Figure 3: Distribution of source localisation error for each dipole. The term 'error' is equivalent to 'variance' in CURRY® software.

The larger number of component that localise the dipole in SII suggest that activity in this region may not time locks to stimulus onset and hence broken down to several distinguish ICs. However, it is difficult to assess the functional role of these components in pain perception since the experiment reported here had only one condition. In future research, comparing these ICs from the dataset, which have different experiment condition, should give the information about the psycho-physiological function of them.

Furthermore, because ICA separate LEP signal into different components, each component assumed to be generated from only one source, the time course activation of each source become available for the entire epoch. In this analysis, all major source dipole appear to peak at around $400 \mathrm{~ms}$. This may be due to inter-regional synchronisation of brain signal as suggested by Varela et al.(2001). The synchronised activation happen at $400 \mathrm{~ms}$ may explain why they are not detectable in standard source localisation method using averaged LEPs. This synchronised phenomenon suggests that the perception of painful stimulus by the subject may be established when the activation of different brain source are integrated at certain time after the laser stimulus.

\section{CONCLUSION}

For a long time, the study of pain-related brain processes (spatially) using PET and $f M R I$, and study (temporally) using LEP have been developed separately. This study demonstrates that by applying ICA to single trial LEP prior to do source localisation, the LEP source dipoles location were consistence with the finding using imaging technique ( $f M R I$ and PET) in other research. Although this result did not verify that the LEP source dipole and the source found in $f \mathrm{MRI}$ and PET are the equivalent, this result has provided the promising solution to investigate the interactive between different imaging modalities.

\section{ACKNOWLEDGEMENT}

Reference:

Bell, A.J., Senowski, T.J., 1995. An informationmaximization approach to blind separation and blind deconvolution. Neural Computation. 7, 11291159.

Bentley, D.E., Derbyshire, S.W.G., Youell, P.D., Jones, A.K.P., 2003. Caudal cingulate cortex involvement in pain processing: an inter-individual laser evoked potential source localisation study using realistic head models. Pain(in press).

Bromm, B., Lorenz, J., 1998. Neurophysiological evaluation of pain. Electroenceph. clin. Neurophysiol. 107, 227-253.

Coghill, R.C., Sang, C.N., Maisog, J.H., Iadarola, M.J., 1999. Pain intensity processing within the human brain: A bilateral, distributed mechanism. Journal of Neurophysiology. 82(4), 1934-1943.

Compumedics CURRY® Multimodal Neuroimaging, ver. 4.5. .

Cuffin, B., 1998. EEG dipole source localization. IEEE Engineering in Medicine and Biology. 17, 118-122.

Derbyshire, S., 2000. Exploring the pain "neuromatrix". Curr Rev Pain. 4, 467-477.

Derbyshire, S.W.G., Jones, A.K.P., Gyulai, F., Clark, S., Townsend, D., Firestone, L.L., 1997. Pain processing during three levels of noxious stimulation produces differential patterns of central activity. Pain. 73, 431-445.

Frot, M., Garcia-Larrea, L., Guenot, M., Mauguiere, F., 2001. Responses of the supra-sylvian (SII) cortex in humans to painful and innocuous stimuli: A study using intra-cerebral recordings. Pain. 94(1), 65-73.

Jung, T.-P., Makeig, S., Westerfield, M., Townsend, J., Courchesne, E., Sejnowski, T.J., 2001. Analysis and Visualization of Single Trial Event-Related Potentials. Human Brain Mapping. 14, 166-185.

Kanda, M., Nagamine, T., Ikeda, A., Ohara, S., Kunieda, T., Fujiwara, N., Sawamoto, N., Matsumoto, R., Taki, W., Shibasaki, H., 2000. primary somatosensory cortex is actively involved in pain processing in human. Brain research. 853, 282-289.

Lotze, M., Wietek, B., Birbaumer, N., Ehrhardt, J., Grodd, W., Enck, P., 2001. Cerebral Activation during Anal and Rectal Stimulation. NeuroImage. 14(5), 1027-1034.

Mai, J.K., Assheuer, J., Paxinos, G. (1997). Atlas of the Human Brain: Academic Press.

Makeig, S., Westerfield, M., Jung, T.-P., Enghoff, S., Townsend, J., Courchesne, E., Sejnowski, T.J., 2002. Dynamic Brain Sources of Visual Evoked Responses. Science. 295, 690-694. 
Mosher, J., Spencer, M., Leahy, R., Lewis, P., 1993. Error bounds for EEG and MEG dipole source localisation. Electroecephal. Clin. Neurophysiol. 86, 303-321.

Nakamura, Y., Paur, R., Zimmermann, R., Bromm, B., 2002. Attentional modulation of human pain processing in the secondary somatosensory cortex: a magnetoencephalographic study. Neuroscience Letters. 328, 29-32.

Niddam, D.M., Yeh, T.C., Wu, Y.T., Lee, P.L., Ho, L.T., Arendt-Nielsen, L., Chen, A.C.N., Hsieh, J.C., 2002. Event-related functional MRI study on central representation of acute muscle pain induced by electrical stimulation. Neuroimage. 17(3), 14371450.

Ninomiya, Y., Kitamura, Y., Yamamoto, S., Okamoto, M., Oka, H., Yamada, N., Kuroda, S., 2001. Analysis of pain-related somatosensory evoked magnetic fields using the MUSIC (multiple signal classification) algorithm for magnetoencephalography. Neuroreport. 12(8), 1657-1661.

Peyron, R., Frot, M., Schneider, F., Garcia-Larrea, L., Mertens, P., Barral, F.G., Sindou, M., Laurent, B., Mauguiere, F., 2002. Role of Operculoinsular
Cortices in Human Pain Processing: Converging Evidence from PET, $f$ MRI, Dipole Modeling, and Intracerebral Recordings of Evoked Potentials. NeuroImage. 17(3), 1336-1346.

Peyron, R., Laurent, B., Garcia-Larrea, L., 2000. Functional imaging of brain responses to pain. A review and meta-analysis (2000). Neurophysiologie Clinique/Clinical Neurophysiology. 30(5), 263-288.

Tarkka, I., Treede, R.-D., 1993. Equivalent electrical source analysis of pain-related somatosensory evoked potentials elicited by a $\mathrm{CO} 2$ laser. Clin Neurophysiol. 10(4), 513-519.

Varela, F., Lachaux, J.P., Rodriguez, E., Martinerie, J., 2001. The brainweb: Phase synchronization and large-scale integration. Nature Reviews Neuroscience. 2(4), 229-239.

Wang, J., 1994. MNLS inverse discriminates between neuronal activity on opposite walls pf a simulated sulcus of the brain. IEEE Trans. Bio. Med. Engr. 41, 470-479.

Watanabe, S., Kakigi, R., Koyama, S., Hoshiyama, M., Kaneoke, Y., 1998. Pain processing traced by magnetoencephalography in the human brain. Brain Topography. 10(4), 255-264. 